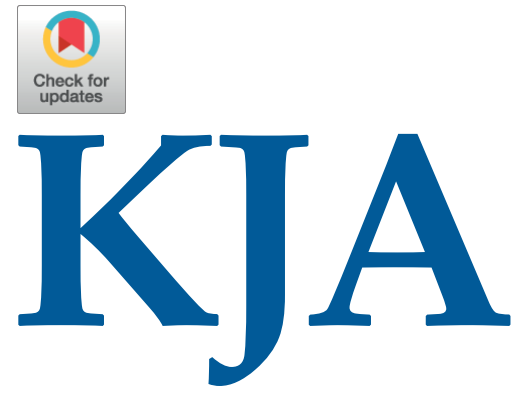

Korean Journal of Anesthesiology

\section{Corrigendum}

Korean J Anesthesiol 2020;73(3):268

https://doi.org/10.4097/kja.19497.e1

pISSN 2005-6419• elSSN 2005-7563

\title{
Tips for troublesome sample-size calculation
}

\section{Junyong In $^{1}$, Hyun Kang ${ }^{2}$, Jong Hae Kim ${ }^{3}$, Tae Kyun Kim ${ }^{4}$, Eun Jin $\mathrm{Ahn}^{5}$, Dong Kyu Lee ${ }^{6}$, Sangseok Lee ${ }^{7}$, Jae Hong Park ${ }^{8}$}

Department of Anesthesiology and Pain Medicine, ${ }^{1}$ Dongguk University Ilsan Hospital, Goyang, ${ }^{2}$ Chung-Ang University College of Medicine, Seoul, ${ }^{3}$ Daegu Catholic University School of Medicine, Daegu, ${ }^{4}$ Yangsan Hospital, Pusan National University School of Medicine, Busan, ${ }^{5}$ Inje University Seoul Paik Hospital, Inje University College of Medicine, ${ }^{6}$ Guro Hospital, Korea University School of Medicine, ${ }^{7}$ Sanggye Paik Hospital, Inje University College of Medicine, Seoul, ${ }^{8}$ Inje University Haeundae Paik Hospital, Inje University College of Medicine, Busan, Korea

Korean Journal of Anesthesiology 2020; 73(2):114-120

https://doi.org/10.4097/kja.19497

The article by In J, et al. entitled, “Tips for troublesome sample-size calculation.” contained an error in the Equation 12.

Before correction:

Adjusted sample size $=\frac{\text { Calculated sample size }}{1-\text { dropout rate }}=\frac{39}{1-0.9}=43.33$

(Equation 12)

The correct information is found below:

Adjusted sample size $=\frac{\text { Calculated sample size }}{1-\text { dropout rate }}=\frac{39}{1-0.1}=43.33$

(Equation 12)

The value for dropout rate was incorrectly printed as 0.9 . The value for dropout rate should be corrected to 0.1 .

The authors apologize for any inconvenience this mistake may have caused.
(C) The Korean Society of Anesthesiologists, 2020

(c) This is an open-access article distributed under the terms of the Creative Commons Attribution Non-Commercial License (http://creativecommons.org/licenses/ by-nc/4.0/), which permits unrestricted non-commercial use, distribution, and reproduction in any medium, provided the original work is properly cited. 